\title{
Long Non-Coding RNA FAM157C Contributed to Clonal Proliferation in Paroxysmal Nocturnal Hemoglobinuria
}

\section{Honglei Wang}

Tianjin Medical University General Hospital https://orcid.org/0000-0002-1023-4826

Hui Liu

Tianjin Medical University General Hospital

\section{Yingying Chen}

Tianjin Medical University General Hospital

\section{Liyan Li}

Tianjin Medical University General Hospital

\section{Zhaoyun Liu}

Tianjin Medical University General Hospital

\section{Lijuan Li}

Tianjin Medical University General Hospital

\section{Shaoxue Ding}

Tianjin Medical University General Hospital

\section{Kai Ding}

Tianjin Medical University General Hospital

Rong Fu ( $\nabla$ florai@sina.com )

Tianjin Medical University General Hospital https://orcid.org/0000-0002-9928-9224

\section{Research article}

Keywords: Paroxysmal Nocturnal Hemoglobinuria, LncRNAs, clone proliferation, FAM157C

Posted Date: January 18th, 2021

DOl: https://doi.org/10.21203/rs.3.rs-147083/v1

License: (c) (i) This work is licensed under a Creative Commons Attribution 4.0 International License. Read Full License 


\section{Abstract}

Background: Paroxysmal nocturnal hemoglobinuria $(\mathrm{PNH})$ is a rare clonal disease of hematopoietic stem cells. However, the mechanism of proliferative advantage of PNH clone is unclear. Long noncoding RNAs (LncRNAs) have a wide range of biological functions, including regulation of gene expression, cell differentiation, and proliferation, while its role in $\mathrm{PNH}$ remains unclear.

Methods: In our study, $\mathrm{CD} 59^{\square}$ and $\mathrm{CD} 59^{+}$granulocytes and monocytes from $5 \mathrm{PNH}$ patients were sorted, and LncRNAs and mRNAs were detected by RNA sequencing. The proliferation-related NF-KB pathway was focused on. A total of 8 mRNAs and 5 LncRNAs were verified by qRT-PCR, and analyzed the correlation with clinical data. Meanwhile, the function of LncRNA was studied.

Results: LncRNA FAM157C were verified to be upregulated in PNH clone cells, which were positively correlated with LDH level and CD59 ${ }^{-}$granulated and monocytes cells ratio. After knockdown of FAM157C gene in PIGA-KO-THP-1 cell line, we found that the cells were blocked in G0/G1 phase and S phase, and the apoptosis rate increased, while the proliferation ability decreased.

Conclusions: LncRNA FAM157C was proved to promote PNH clone proliferation, which is the first time to explore the role of LncRNAs in PNH.

\section{Introduction}

Paroxysmal nocturnal haemoglobinuria ( $\mathrm{PNH}$ ) is an acquired clonal disorder of haematopoietic stem cells (HSCs) caused by somatic mutation of phosphatidylinositol glycan A gene (PIG-A) on chromosome Xp22.1. PIGA mutation leads to impaired glycosylphosphatidylinositol (GPI) synthesis, resulting in the loss of GPI anchored protein (GPI-AP) on the cell surface (such as CD59, CD55), resulting in the destruction of the increased sensitivity of the blood cells to the activation of complement, and the main clinical manifestations are chronic intravascular hemolysis, bone marrow failure, high risk of thrombosis, etc ${ }^{12}$. The PIG-A mutation alone is necessary but insufficient to explain PNH clonal expansion. PNH clones do not self-renew and survive for only 3-4 months ${ }^{4}$. In order to cause PNH, PIGA mutations must achieve proliferative advantages. However, the mechanism of proliferative advantage of PNH clone is not clear until now.

Long noncoding RNAs (LncRNAs) range from $200 \mathrm{nt}$ up to $~ 100 \mathrm{~kb}$ in length which do not encode proteins and are located in the nucleus or cytoplasm ${ }^{56}$. It is now well recognized that more than $75 \%$ of the human genome is functional and encodes large numbers of LncRNAs ${ }^{7}$. LncRNAs have a wide range of biological functions, including regulation of gene expression, such as chromosome dosage compensation, imprinting, epigenetic regulation, nuclear transport, transcription, mRNA splicing and translation; as well as cell differentiation, cell proliferation and substance metabolism ${ }^{89}$. In the past decade, the abnormal expression of LncRNAs have been proved to be involved in the pathogenesis of many diseases, including tumors, metabolic diseases and cardiovascular diseases ${ }^{101112}$. At present, studies on the mechanism of PNH clone proliferation are mainly focused on protein-coding genes, the function and clinical significance of LncRNAs in the PNH remain unknown. The purpose of this study was to investigate the role of LncRNAs in PNH clone proliferation. 


\section{Methods}

\subsection{Patients and clinical samples}

A total of $35 \mathrm{PNH}$ patients were enrolled in our study, who were diagnosed in the Hematology Department of Tianjin Medical University General Hospital from November 2017 to August 2019 according to international PNH Study Group Criteria ${ }^{13}$. LncRNAs and mRNAs were detected and analysed in 5 PNH patients. Then, selected LncRNAs and mRNAs expressions were verified in other $30 \mathrm{PNH}$ patients, and the correlation were analyzed with clinical indexes, including hemoglobin $(\mathrm{Hb})$, white blood cells $(\mathrm{WBC})$, platelet $(\mathrm{PLT})$, reticulocyte (Ret), lactate dehydrogenase (LDH), total bilirubin (TBIL), free hemoglobin, haptoglobin and PNH clone. Their clinical features were shown in Table 1.

Table 1

Clinical characteristics of all PNH patients

\begin{tabular}{|lll|}
\hline Characteristics & Sequencing group & Validation group \\
\hline Total no. of patients & 5 & 30 \\
\hline Gender M/F & $3 / 2$ & $19 / 11$ \\
\hline Age Median (range) & $38(16-62)$ & $41(16-68)$ \\
\hline Clinical classification n (\%) & & \\
\hline classical PNH & $3(60.00)$ & $22(73.33)$ \\
\hline PNH-AA & $2(40.00)$ & $8(26.67)$ \\
\hline History of thrombosis n (\%) & 0 & $11(36.67)$ \\
\hline HGB (g/L) & $76.60 \pm 28.29$ & $79.71 \pm 23.97$ \\
\hline Ret (\%) & $11.79 \pm 7.44$ & $8.285 \pm 5.589$ \\
\hline WBC (*109/L) & $7.49 \pm 3.53$ & $5.47 \pm 3.29$ \\
\hline PLT (*109/L) & $149.00 \pm 159.20$ & $116.00 \pm 95.03$ \\
\hline TBIL (umol/L) & $24.48 \pm 14.69$ & $34.53 \pm 18.59$ \\
\hline LDH (U/L) & $2236.00 \pm 1498.0$ & $1543.00 \pm 1181.0$ \\
\hline Granulocyte CD59- (\%) & $96.41 \pm 2.458$ & $74.90 \pm 24.39$ \\
\hline Erythrocyte CD59- $(\%)$ & $74.12 \pm 21.42$ & $47.50 \pm 31.77$ \\
\hline
\end{tabular}

Our study confirmed "International ethical guidelines for biomedical research involving human subjects (2002)" developed by Council for International Organizations of Medical Sciences (CIOMS) in collaboration with World Health Organization (WHO) and was approved by the Ethical Committee of the Tianjin Medical University.

\subsection{Cell sorting by flow cytometry}


Ten milliliter peripheral blood were taken in ethylenediaminetetraacetic acid (EDTA) anticoagulant tube from $\mathrm{PNH}$ patients. Firstly, the supernatant was discarded after centrifugation ( $3 \mathrm{~g}, 5$ minutes), and the remaining blood was separated into five centrifugal tubes. After 15 minutes, the erythrocyte lysate was centrifuged again and washed with PBS. Anti-CD59 FITC (BD PharMingen, USA) was added for 20 min in dark room. Furthermoer, to obtain $\mathrm{CD} 59^{-}$and $\mathrm{CD} 59^{+}$granulocytes and monocytes, $\mathrm{PNH}$ clone was sorted by Flow cytometry (BD FACSAria囚, USA). The separation purity is above $90 \%$ (Fig. 1A, B).

\subsection{RNA sequencing and analysis}

After obtain CD59-and $\mathrm{CD} 59^{+}$granulocytes and monocytes, quality of total RNA was extracted from PNH patients. RNA-seq analysis was performed by Beijing Novogene Bioinformatics Technology Co., LTD (Appendices 1).

\section{4 qRT-PCR}

Total RNA was extracted from CD59“cells by RNeasy kit (Takara Bio Inc, RR420A, Japan). cDNA was synthesized from $1 \mu \mathrm{g}$ of total RNA using a reverse transcription kit (Takara, Japan) and purified with the QIAquick PCR Purification Kit (Qiagen). Quantitative PCR (qRT-PCR) was performed in duplicates with QuantiTect SYBR Green PCR Kit (Takara, Tli RNaseH Plus) with a Light Cycler 1.5 Real-time PCR machine (Roche, Indianapolis, IN, USA). Specific primers designed to amplify fragment cross-exons of these genes which included TAB2, TLR4, LYN, CFLAR, TNFAIP3, PTGS2, TRIM25, CXCL8, LINC01002 and FAM157C are detailed in Table 2. The BIO-RAD CFX MANAGER software was used to analyze the melting curve and the amplification curve (Quantitative curve), and the $\mathrm{Ct}$ values of each group were read. The relative quantitative multiplier of each group (relative fold, RF) was expressed by $2-\Delta \Delta C$ t value and used for statistical analysis. 
Table 2

Gene primer sequences

\begin{tabular}{|llll|}
\hline gene & forword & reverse & $\begin{array}{c}\text { annealing } \\
\text { temperature }\end{array}$ \\
\hline LINC01002 & TCCAGTCAGCCTCTACAGACCAAG & GATACGATGGAGCTGTGCCTGTG & 57.0 \\
\hline FAM157C & AAGACGGAGCAGCACAGTCATTC & TGTTCGGACAGTTACACGCCATG & 60.8 \\
\hline CFLAR & GCTGATGGCAGAGATTGGTGAGG & TCCAACTCAACCACAAGGTCCAAG & 57.1 \\
\hline LYN & AGGCTCTACGCTGTGGTCACC & TTGCCACCTTCATCGCTCTTCAG & 56.0 \\
\hline CXCL8 & TCTCTTGGCAGCCTTCCTGA & TTTCTGTGTTGGCGCAGTGT & 57.1 \\
\hline TNFAIP3 & CTGCTGGCTGCCTGTCTCAAG & GTTCTGGAACCTGGACGCTGTG & 56.5 \\
\hline PTGS2 & TGGTCTGGTGCCTGGTCTGATG & CCTGCTTGTCTGGAACAACTGCTC & 57.1 \\
\hline TLR4 & GAGGCAGCTCTTGGTGGAAGTTG & CAAGCACACTGAGGACCGACAC & 56.0 \\
\hline TRIM25 & CTGGTGCGTGGAGTGGTTCAAC & TGTCGGCAACAGCGAAGAAGATG & 56.5 \\
\hline Table2 & ACCTCCAGCACTTCCTCTTCAGTC & TGTTCATCTCCTGTGGCAGCATTC & 56.0 \\
\hline GAPDH & CAGGAGGCATTGCTGATGAT & GAAGGCTGGGGCTCATTT & \\
\hline
\end{tabular}

\subsection{Cell line and cell culture}

The Cas9 and sgRNA overexpressed lentivirus were constructed to infect THP-1 cells, and then the GFP (Cas9 vector) and mCherry (sgRNA vector) positive cells were screened by flow cytometry. The monoclonal cells were selected and amplified after identification, finally the PIGA gene knock-down (PIGAKO) monoclonal cell line were obtained. The PIGA-KO-THP-1 cells were cultured in RPMI-1640 + 10\% fetal bovine serum (Gibco) + $1 \%$ double antibody. PIGA-KO cell clones were established based on and mutations as assessed by PCR, gene sequencing and their phenotypes (loss of FLAER and CD59 positive staining). The cells were cultured in a constant temperature incubator at $5 \% \mathrm{CO} 2$ and $37^{\circ} \mathrm{C}$, and were passed through at a ratio of $1: 3$ every 48 hours.

\subsection{Lentivirus transfection}

Lentivirus vector expressing an shRNA against the FAM157C were used for transfection of PIGA-Ko-THP-1 cells for $72 \mathrm{~h}$. The experiment was divided into three groups: the control group, the empty virus group, and the FAM157C knock-down group. $5 \times 10^{4}$ cells were inoculated into 24 -well plates, with a final volume of $500 \mathrm{ul}$, incubated at $37^{\circ} \mathrm{C}$ and $5 \% \mathrm{CO} 272 \mathrm{~h}$. The transfection rate and knock down rate were evaluated by Flow cytometry and qRT-PCR. They have obtained stable transfectant.

\subsection{CCK-8 assay}

For measurement of cell proliferation, we utilized the CCK-8 kit (Dojindo Laboratories) to perform CCK-8 assay: Cells were seeded on a 96-well plate and incubated in CCK-8 solution for 4 hours at $37^{\circ} \mathrm{C}, 5 \% \mathrm{CO} 2$ incubator followed by measurement of the absorbance at wavelength of $450 \mathrm{~nm}$. 


\subsection{Cell apoptosis assay}

Apoptosis was determined by translocation of phosphatidylserine to the cell surface using an Annexin V-PE and 7-ADD apoptosis detection kit (BD PMG). The stable knockdown LncRNA FAM157C PIGA-KO-THP-1 cells and its negative control cells were harvested and washed twice in cold PBS, and re-suspended in Annexin VPE and 7-ADD for 30 min in the dark. Cell apoptosis was analyzed by using Cell Quest software on a Flow Cytometer (Beckman).

\subsection{Cell cycle analysis}

The cells were first harvested after 72 hours of transfection and the cell suspension was then digested. Afterwards, the cells were fixed with ethanol $(70 \%)$ for $4 \mathrm{~h}$ at $4{ }^{\circ} \mathrm{C}$ and the supernatant was then discarded, followed by incubation with an RNA enzyme containing iodide (PI, BD PMG). After the cells were washed with PBS three times, the cell cycle was detected by using Flow Cytometer (Beckman), and data analysis was conducted through Kaluza. The experiment was repeated three times.

\subsection{Statistical analysis}

GraphPad Prism5 statistical software was used for statistical analysis. Results were expressed as mean \pm standard deviations. The independent sample mean comparison had been done using the t-test (for data with normal distribution) and nonparametric test (for data without normal distribution). Spearman's correlation analysis was used to evaluate the association between qualitative variables. A value of $P<0.05$ was considered statistically significant.

\section{Results}

\subsection{Some abnormal LncRNAs and mRNAs were sorted by RNA-seq in PNH clone}

For transcriptome profiling of $\mathrm{CD} 59^{-}$cells and $\mathrm{CD} 59^{+}$cells in PNH patients, RNA-seq was performed in CD $59^{\square}$ and $\mathrm{CD} 59^{+}$granulocytes and monocytes from $5 \mathrm{PNH}$ patients screening. Transcription analysis revealed that 742 upregulated and 1376 downregulated LncRNAs, and 3276 upregulated and 213 downregulated mRNAs (Fig. 2A, B).

The 173 upregulated mRNAs which FPKM $>5$ and over 3 patients were screened. Then we screened 30 mRNAs related to proliferation, apoptosis and thrombosis. The 7 upregulated LncRNAs which FPKM $>5$ and over 3 patients were screened (Appendices 2). The expression of upregulated mRNAs and LncRNAs in $5 \mathrm{PNH}$ patients were showed by venn diagrams (Fig. 2C).

By KEGG pathway enrichment analysis, putative gene network interactions of differentially expressed genes were significantly enriched for pathway of TNF signaling pathway, NF-kappa B signaling pathway, Neurotrophin signaling pathway and so on. We focused on the proliferation relative pathway-NF-kB pathway (Fig. 2D). The mRNA which FPKM >10 and over 3 patients were selected to search out the upstream regulation LncRNAs. A total of 8 mRNAs (TAB2, TLR4, LYN, CFLAR, TNFAIP3, PTGS2, TRIM25, CXCL8) were 
screened out, and upstream regulated LncRNAs (LINC01002, FAM157C, CTD-2530H12.2, XLOC-064331, XLOC-106677) were found by co-expression method (Fig. 2E). Only LINC01002 and FAM157C were designed the appropriate primer sequence. The 8 mRNAs and 5 LncRNAs were upregulated in CD59-granulocytes and monocytes.

\subsection{LncRNA FAM157C were verified to be upregulated by qRT- $\mathrm{PCR}$, and correlated with hemolysis indicators}

The expressions of some abnormal LncRNAs and mRNAs in $30 \mathrm{PNH}$ patients were detected by qRT-PCR. As the results of qRT-PCR showed, LncRNA FAM157C $(11.530 \pm 6.628)$ expressions in PNH clone was consistently higher than that $(5.482 \pm 6.785, p=0.0055)$ in $\mathrm{CD}_{59}{ }^{+}$cells from $30 \mathrm{PNH}$ patients (Fig. $\left.3 \mathrm{~A}\right)$. LncRNA LINC01002 expression was $(6.118 \pm 10.08)$ in PNH clone, no significant difference compared with CD59 ${ }^{+}$cells $(4.883 \pm 9.208, p=0.6374)$ (Fig. 3B). While there were no significant differences in mRNAs (Fig. 3C, Table 3).

Table 3

Comparison of mRNAs expression

\begin{tabular}{|lllll|}
\hline mRNA & CD59-granulocytes and monocytes & CD59 $^{+}$granulocytes and monocytes & $\mathbf{n}$ & $P$ value \\
\hline LYN & $1.373 \pm 1.676$ & $1.957 \pm 2.956$ & 30 & 0.4679 \\
\hline TLR4 & $2.256 \pm 1.928$ & $6.213 \pm 15.84$ & 30 & 0.3001 \\
\hline Table 2 & $1.496 \pm 1.569$ & $2.989 \pm 5.373$ & 30 & 0.2821 \\
\hline PTGS2 & $1.963 \pm 2.491$ & $2.336 \pm 3.475$ & 30 & 0.4462 \\
\hline CXCL8 & $4.606 \pm 7.600$ & $6.423 \pm 11.55$ & 30 & 0.2228 \\
\hline CFLAR & $3.091 \pm 2.126$ & $3.128 \pm 3.872$ & 30 & 0.9662 \\
\hline TRIM25 & $1.253 \pm 0.8713$ & $2.846 \pm 5.872$ & 30 & 0.2677 \\
\hline TNFAIP3 & $3.426 \pm 4.189$ & $5.769 \pm 8.517$ & 30 & 0.1095 \\
\hline
\end{tabular}

The correlation between the expression of LncRNA FAM157C and the clinical indexes (blood routine, reticulocyte, hemolysis indicators) were analyzed. The high expression of FAM157C were positively correlated with LDH level and $(r=0.4156, p=0.0224)$ and $C^{2} 59^{-}$granulated and monocytes cells ratio. $(r=0.4793, p=$ 0.0074) (Fig. 3D).

\subsection{Knock-down of LncRNA FAM157C inhibited the proliferation of PIGA-KO-THP-1 cells}

The verification results of PIGA-KO-THP-1 cells are shown in the Fig. 4A, B, C. LncRNA FAM157C was knocked down by lentivirus with a transfection rate of $70 \%$ and a knock-down rate of $90 \%$. The cell viability (\%) of the control group, empty virus group and LncRNA FAM157C knock-down group were (100 \pm 0$)$, $(95.20 \pm 3.178)$ and $(91.93 \pm 5.423)$ after $24 \mathrm{~h}$ transfection respectively. There was no statistical difference between the three groups $(p=0.1807)$. While at $48 \mathrm{~h}$ and $72 \mathrm{~h}$, the cell viability of three groups were $(100 \pm 0)$ vs $(93.75 \pm 5.995)$ 
vs $(77.49 \pm 6.597)$ and $(100 \pm 0)$ vs $(92.795 \pm 5.802)$ vs $(60.47 \pm 2.059)$. The viability of the FAM157C knockdown group was significant lower than those of control and empty virus group $(p=0.0275,0.0388,0.0009$, 0.0052). (Fig. 5A).

Meanwhile, the apoptosis rate increased after transfection of lentivirus FAM157C. The apoptosis rate of the FAM157C knock-down group (6.256 \pm 0.5453$) \%$ was significant higher than the control group (2.483 \pm $0.3083) \%$ and empty virus group $(2.926 \pm 0.5517) \%(p=0.0138,0.0066)$ at $24 \mathrm{~h}$. There was no statistical difference between empty virus group and control group $(p=0.0899)$. The similar results were found at $48 \mathrm{~h}$ and $72 \mathrm{~h}$. The apoptosis rate of three groups were $(5.593 \pm 0.6400) \%,(6.723 \pm 0.3256) \%$, and $(11.30 \pm$ $1.075) \%$ at $48 \mathrm{~h}(p=0.0137,0.0217)$, and $(9.797 \pm 0.3235) \%,(10.21 \pm 0.3005) \%$, and $(18.81 \pm 0.5363) \%$ at $72 \mathrm{~h}(p=0.0012,0.00087)$. (Fig. 5B, C).

We also observed the cell cycle, and the results showed that the proportion of G0/G1 phase and S phase increased while the proportion of G2 phase decreased, indicating the cells were blocked in G0/G1 phase and $\mathrm{S}$ phase. The G0/G1 phase of the control group, empty virus group and FAM157C knockdown group were $(62.98 \pm 1.513) \%(65.95 \pm 1.174) \%$ and $(70.00 \pm 0.2404) \%$, S phase were $(3.825 \pm 0.7849) \%,(5.920 \pm 0.9192) \%$ and $(13.47 \pm 1.039) \%$, G2 phase were $(32.81 \pm 1.612) \%(27.47 \pm 1.160) \%$ and $(16.54 \pm 0.7990) \%$ after transfection, in which were statistically different $(p=0.0269,0.0198,0.0145)$. (Fig. 5D, E).

\section{Discussion}

In recent years, studies have found that PIG-A mutations can also be detected in normal people, accounting for about $10 \%$. However, no PNH clones and proliferation have been found, and no clinical symptoms of PNH 14. In Shin TH et al 2019, the data gathered about PNH macaque model using CRISPR/Cas9 technology to create model of hematologic disease based on the near phylogenetic/functional similarity between macaque and human was shown that there was no intrinsic clonal amplification of PNH-HSPCs ${ }^{15}$. At present, studies reported that immune-escape characteristics ${ }^{16} 1718$, anti-apoptotic properties ${ }^{19} 20$, and second gene mutations 212223 may be involved in the amplification of PNH clones. T-lymphocyte immune attack $\mathrm{GPI}^{+} \mathrm{HSC}$, and $\mathrm{GPI}^{-} \mathrm{HSC}$ is less vulnerable to attack. Studies have found that PNH cells are protected from NK/T effector cells due to the lack of GPI-anchored cytomegalovirus ul-16 binding protein (ULBPs) or CD1d restriction 2425 . CD109 has been reported to be a protein in GPI-APs, a TGF-co-receptor, which plays a key role in inhibiting TGF-signal-mediated erythrocyte differentiation. The lack of CD109 may make PIGA-mutated HSPCs more sensitive to TGF- $\beta$, leading to more easy differentiation of mutant erythroid progenitors into mature erythrocytes ${ }^{26}$. The studies found that PNH clone cells have anti-apoptotic properties. In addition, Bcl-2, Bcl-XL, Bag-1, McL-1 and other anti-apoptotic genes were significantly increased in PNH patients, and played an important role in the anti-apoptotic process ${ }^{27}$. The studies also found that PIG-A mutations disrupt lipid raft formation of cell membrane, this change passivation for promoting apoptosis signals or growth inhibition ${ }^{28}$. The theory of secondary genetic mutations has been reported since the $1970 \mathrm{~s}^{21}$. Additional mutant genes such as HMGA2 2930 , WT1 ${ }^{31}$, TET2 ${ }^{32}$ and RBPJ ${ }^{33}$ have been reported in PNH patients. Most cases of PNH can carry additional mutations and these mutations are secondary strikes. PIGA mutation is the initial mutation, the nature of $\mathrm{PNH}$ is a single gene disease, and its clinical manifestations are mainly 
determined by PIGA mutations rather than myeloid gene mutations ${ }^{34}$. However, various theories cannot explain all the pathological mechanisms, and PNH clones must be involved in other mechanisms to gain proliferation advantage in patients.

Although LncRNAs do not encode proteins, they play an important role in cell proliferation and differentiation, and are widely studied in oncologic diseases. Not only solid tumors, but also non-solid tumors and even autoimmune diseases have been studied. The LncRNAs LOC101928834, H19, WT1-AS, TCL6, LEF1-AS1, EPB41L4A-AS1, PVT1, GAS5 and ZFAS were found relevant to (myelodysplastic syndromes (MDS) pathogenesis and outcome ${ }^{35} 36$. Many LncRNAs such as MALAT1, GAS5, DLEU2, H19 and so on were reported in diagnosis ang progression of multiple myeloma (MM) ${ }^{37} 38$. The LncRNAs HOTAIR, LincRNA-p21, LncRNA H19 and MALAT1 play important role in clinical diagnosis ang progression of Rheumatoid arthritis (RA) ${ }^{39}$. The mechanism of action of LncRNA is complex. LncRNAs can interact with DNA, RNA, or protein. LncRNAs are involved in various pathways, including p53, NF-KB, PI3K/AKT, Notch and so on.

In our study, the results of RNA sequencing in PNH patients showed a large number of differentially expressed LncRNAs and mRNAs, many of which were involved in cell proliferation, thrombosis, etc. Such results gave us a lot of information, LncRNAs may play an important role in PNH clone proliferation. By verification, we found that the level of LncRNA FAM157C in CD59- cells siginificantly increased, and was positively correlated with the LDH levels and CD59- granulated and monocytes cells ratio. After knockdown of FAM157C gene, it was found that the cells were blocked in G0/G1 phase and S phase, the apoptosis rate increased, the proliferation ability decreased. The FAM157C was expressed in bone marrow, spleen and other organs, and its function has not been reported in the literature. The results of our experiment suggested that FAM157C gene may promote PNH clone proliferation. The mechanism remains to be further studied.

\section{Declarations}

\section{Acknowledgements}

This work was supported by the National Natural Science Foundation of China (Grant nos. 81770110, 81900131, 82000128), the Tianjin Municipal Natural Science Foundation (Grant nos. 18JCYBJC27200, 18JCQNJC80400), the Tianjin Education Commission Research Project (2018KJ043, 2018KJ045).

\section{Author contributions}

Rong Fu designed the research and revised the manuscript. Honglei Wang, Hui Liu and Yingying Chen performed the experiments, analyzed the data, and wrote the article. Zhaoyun Liu, Liyan Li, Lijuan Li, Shaoxue Ding and Kai Ding contributed to the experimental work and the collection of patients' features. All authors read and approved the final manuscript.

\section{Conflicts of interest}


All authors report no conflicts of interest.

\section{Data Statement}

The data that support the findings of this study are available from the corresponding author upon reasonable request.

\section{References}

1. Rotoli B, Luzzatto L. Paroxysmal nocturnal hemoglobinuria. Semin Hematol. 1989;26(3):201-7.

2. Hill A, DeZern AE, Kinoshita T, Brodsky RA. Paroxysmal nocturnal haemoglobinuria.Nat Rev Dis Primers. 2017 May 18; 3:17028.

3. Parker CJ. (2016) Update on the diagnosis and management of paroxysmal nocturnal hemoglobinuria. Hematology. American Society of Hematology. Education Program, 2016, 208-216.

4. Brodsky RA. Paroxysmal nocturnal hemoglobinuria. Blood. 2014 Oct 30;124(18):2804-11.

5. Ma Y, Zhang J, Wen L, Lin A. Membrane-lipid associated IncRNA: A new regulator in cancer signaling. Cancer Lett. 2018 Apr;10:419:27.

6. Bhan A, Soleimani M, Mandal SS. Long Noncoding RNA and Cancer: A New Paradigm.Cancer Res. 2017 Aug 1;77(15):3965-3981.

7. Sanfilippo PG, Hewitt AW. Translating the ENCyclopedia Of DNA Elements Project findings to the clinic: ENCODE's implications for eye disease. Clin Exp Ophthalmol. 2014;42:78-83.

8. Yang X, Xie X, Xiao YF, Xie R, Hu CJ, Tang B, et al. The emergence of long non-coding RNAs in the tumorigenesis of hepatocellular carcinoma. Cancer Lett. 2015;360:119-24.

9. Sun T, Wong N. Transforming growth factor-beta-induced long noncoding RNA promotes liver cancer metastasis via RNA-RNA crosstalk. Hepatology. 2015;61:722-4.

10. Chi Y, Wang D, Wang J, Yu W, Yang J. Long Non-Coding RNA in the Pathogenesis of Cancers. Cells. 2019 Sep 1;8(9). pii: E1015.

11. Goyal N, Kesharwani D, Datta M. Lnc-ing non-coding RNAs with metabolism and diabetes: roles of IncRNAs. Cell Mol Life Sci. 2018 May;75(10):1827-37.

12. Uchida S, Dimmeler S. Long noncoding RNAs in cardiovascular diseases.Circ Res. 2015 Feb 13;116(4):737-50.

13. Parker $\mathrm{C}$, Omine M, Richards S. et, al. International PNH Interest Group. Diagnosis and management of paroxysmal nocturnal hemoglobinuria. Blood. 2005;106:3699-709.

14. Hu R, Mukhina GL, Piantadosi S, Barber JP, Jones RJ, Brodsky RA. PIG-A mutations in normal hematopoiesis. Blood. 2005 May 15; 105(10):3848-54.

15. Shin TH, Baek EJ, Corat MAF. so on. CRISPR/Cas9 PIG -A gene editing in nonhuman primate model demonstrates no intrinsic clonal expansion of PNH HSPCs. Blood. 2019 Jun 6;133(23):2542-5.

16. Rotoli B, Luzzatto L. Paroxysmal nocturnal haemoglobinuria. Baillières Clin Haematol 1989 2: 113-138.

17. Karadimitris A, Luzzatto L. The cellular pathogenesis of paroxysmal nocturnal haemoglobinuria. Leukemia. 2001 Aug; 15(8):1148-52. 
18. YoungNS MaciejewskiJP, SloandE, et al. The relationship of aplastic anemia and PNH. Int J Hematol, 2002, vol. 76 suppl 2(pg. 168-172).

19. Brodsky RA, Vala MS, Barber JP, Medof ME, Jones RJ. Resistance to apoptosis caused by PIG-A gene mutations in paroxysmal nocturnal hemoglobinuria. Proc Natl Acad Sci U S A. 1997 Aug 5;94(16):875660.

20. Horikawa K, Nakakuma H, Kawaguchi T, Iwamoto N, Nagakura S, Kagimoto T, Takatsuki K. Apoptosis resistance of blood cells from patients with paroxysmal nocturnal hemoglobinuria, aplastic anemia, and myelodysplastic syndrome. Blood. 1997 Oct 1;90(7):2716-22.

21. Hansen NE, Killmann SA. Paroxysmal nocturnal hemoglobinuria in myelofibrosis. Blood. 1970;36:42831.

22. Brodsky RA. Paroxysmal nocturnal hemoglobinuria: Stem cells and clonality. Hematology Am Soc Hematol Educ Program. 2008:111-115.

23. Tominaga R, Katagiri T, Kataoka K, Kataoka K, Wee RK, Maeda A, Gomyo H, Mizuno I, Murayama T, Ogawa S, Nakao S. Paroxysmal nocturnal hemoglobinuria induced by the occurrence of BCR-ABL in a PIGA mutant hematopoietic progenitor cell. Leukemia. 2016 May;30(5):1208-10.

24. Hanaoka N, Kawaguchi T, Horikawa K, Nagakura S, Mitsuya H, Nakakuma H. Immunoselection by natural killer cells of PIGA mutant cells missing stress-inducible ULBP. Blood. 2006;107(3):1184-91.

25. Gargiulo L, Papaioannou M, Sica M, Talini G, Chaidos A, Richichi B, Nikolaev AV, Nativi C, Layton M, de la Fuente J, Roberts I, Luzzatto L, Notaro R, Karadimitris A. Glycosylphosphatidylinositol-specific, CD1drestricted T cells in paroxysmal nocturnal hemoglobinuria. Blood. 2013 Apr 4;121(14):2753-61.

26. Tanabe Mikoto MD, Nguyen Hoang Maianh MD, Kohei Hosokawa MD, PhD N, Nakagawa MD, Luis Espinoza MD, PhD. Hirohito Yamazaki, MD PhD, Shinji Nakao, MD PhD. A GPI-Anchored Protein, CD109, Protects Hematopoietic Progenitor Cells from Erythroid Differentiation Induced By TGF- $\beta$. Blood (2019) 134 (Supplement-1): 3736.

27. Heeney MM, Ormsbee SM, Moody MA, Howard TA, DeCastro CM, Ware RE. Increased expression of antiapoptosis genes in peripheral blood cells from patients with paroxysmal nocturnal hemoglobinuria. Mol Genet Metab. 2003 Apr;78(4):291-4.

28. El-Sherbiny YM, Kelly RJ, Hill A, Doody GM, Hillmen P, Cook GP. Altered natural killer cell subset homeostasis and defective chemotactic responses in paroxysmal nocturnal hemoglobinuria. Blood. 2013 Sep 12;122(11):1887-90.

29. Inoue N, Izui-Sarumaru T, Murakami Y, et al. Molecular basis of clonal expansion of hematopoiesis in 2 patients with paroxysmal nocturnal hemoglobinuria (PNH). Blood. 2006;108(13):4232-6.

30. Murakami Y, Inoue N, Shichishima T, Ohta R, Noji H, Maeda Y, Nishimura J, Kanakura Y, Kinoshita T. Deregulated expression of HMGA2 is implicated in clonal expansion of PIGA deficient cells in paroxysmal nocturnal haemoglobinuria. Br J Haematol. 2012 Feb;156(3):383-7.

31. Zhang Y, Fu R, Wang Y, Li L, Liu H, Liu C, Zhang T, Ding S, Li L, Ruan E, Qu W, Wang H, Wang X, Wang G, Wu Y, Song J, Liu H, Xing L, Guan J, Shao Z. Abnormal WT1 gene expression in paroxysmal nocturnal hemoglobinuria. Article in Chinese. Zhonghua Xue Ye Xue Za Zhi. 2014 Jul;35(7):596-600. 
32. Lobry C, Bains A, Zamechek LB, Ibrahim S, Aifantis I, Araten DJ. Analysis of TET2 mutations in paroxysmal nocturnal hemoglobinuria (PNH). Exp Hematol Oncol. 2019 Aug;21:8:17.

33. Li L, Liu H, Wang H, Liu Z, Chen Y, Li L, Song J, Wang G, Fu R. Abnormal expression and mutation of the RBPJ gene may be involved in CD59-clonal proliferation in paroxysmal nocturnal hemoglobinuria. Exp Ther Med. 2019 Jun;17(6):4536-46.

34. Awada H, Rahman S, Durrani J. and so on. Leukemia evolving from paroxysmal nocturnal hemoglobinuria. Leukemia. 2020 Jan;34(1):327-30.

35. Li N, Ma Y, Wang W, Yin CC, Wu W, Sun R, Zhao G, Li S, Wang X. LOC101928834, a novel IncRNA in Wnt/ $\beta$-catenin signaling pathway, promotes cell proliferation and predicts poor clinical outcome in myelodysplastic syndromes. Clin Sci (Lond). 2020 Jun 12;134(11):1279-1293.

36. Szikszai K, Krejcik Z, Klema J, Loudova N, Hrustincova A, Belickova M, Hruba M, Vesela J, Stranecky V, Kundrat D, Pecherkova P, Cermak J, Jonasova A, Dostalova Merkerova M. LncRNA Profiling Reveals That the Deregulation of H19, WT1-AS, TCL6, and LEF1-AS1 Is Associated with Higher-Risk Myelodysplastic Syndrome. Cancers (Basel). 2020 Sep 23;12(10):2726.

37. Ronchetti D, Agnelli L, Taiana E, Galletti S, Manzoni M, Todoerti K, Musto P, Strozzi F, Neri A. Distinct IncRNA transcriptional fingerprints characterize progressive stages of multiple myeloma. Oncotarget. 2016;7:14814-30.

38. Pan Y, Chen H, Shen X, Wang X, Ju S, Lu M, Cong H. Serum level of long noncoding RNA H19 as a diagnostic biomarker of multiple myeloma. Clin Chim Acta. 2018;480:199-205.

39. Li Z, Li X, Jiang C, Qian W, Tse G, Chan MTV, Wu WKK. Long non-coding RNAs in rheumatoid arthritis. Cell Prolif. 2018 Feb;51(1):e12404.

\section{Figures}


A
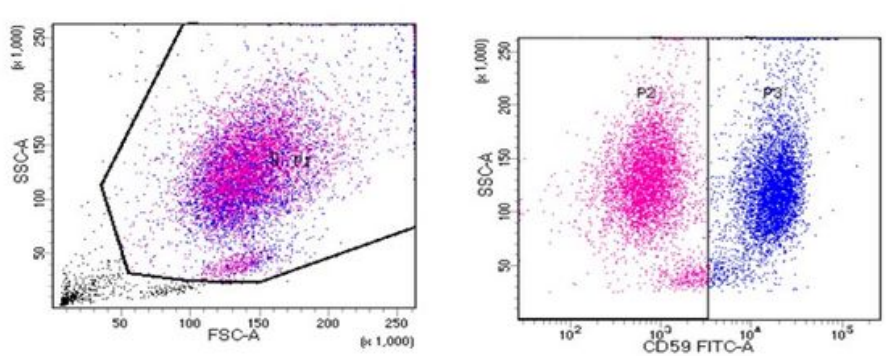

B
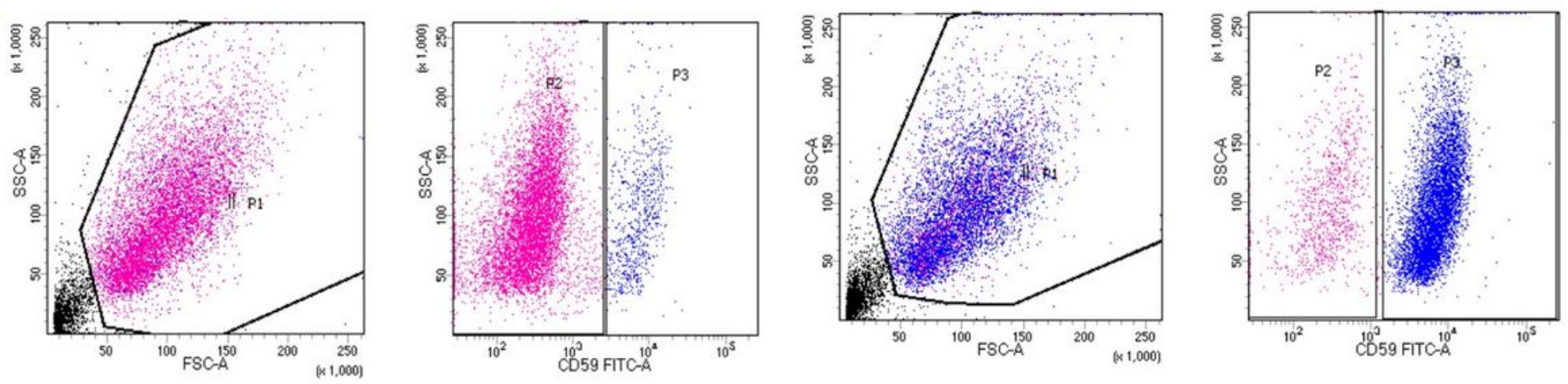

\begin{tabular}{|l|r|r|r|}
\hline Tube: CD59- & & & \\
Population & \#Events & \% Parent & $\%$ Total \\
\hline All Events & 10.000 & \%\#H & 100.0 \\
\hline P1 & 8.156 & 81.6 & 81.6 \\
\hline P3 & 732 & 9.0 & 7.3 \\
\hline P2 & 7.416 & 90.9 & 74.2 \\
\hline
\end{tabular}

\begin{tabular}{|lr|r|r|}
\hline Tube: CD59+ & & & \\
Population & \#Events & \% Parent & \%Total \\
\hline All Events & 10.000 & \#\#\# & 100.0 \\
\hline$\square$ P1 & 7.224 & 72.2 & 72.2 \\
\hline P3 & 6.292 & 87.1 & 62.9 \\
\hline P2 & 919 & 12.7 & 9.2 \\
$\square$ P4 & 0 & 0.0 & 0.0 \\
\hline
\end{tabular}

\section{Figure 1}

: The cells of PNH patients were sorted by Flow cytometry to obtain CD59『and CD59+ granulocytes and monocytes. B: Sorting purity of the CD59『and CD59+ granulocytes and monocytes. The sorting purity is about $90 \%$. 
A

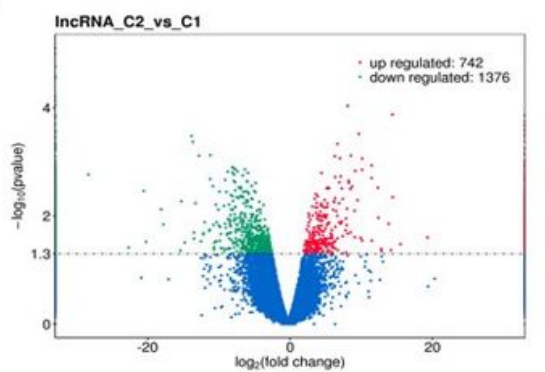

B

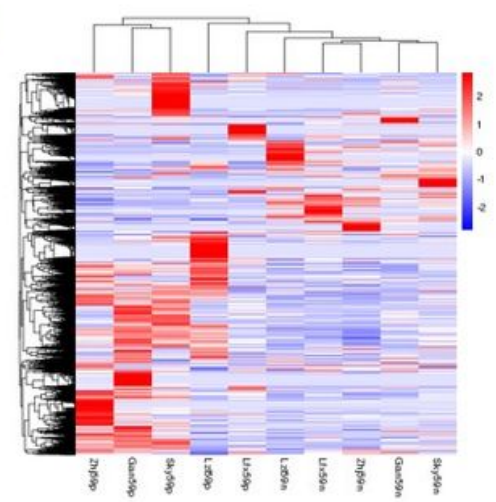

C

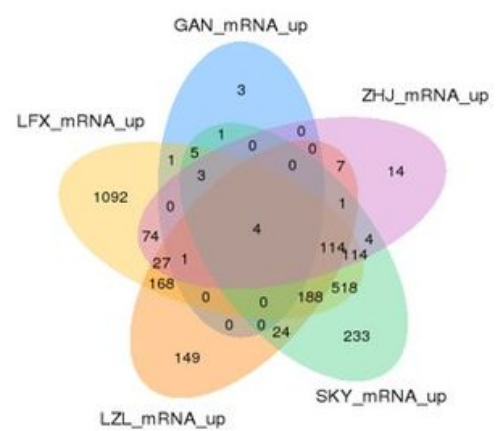

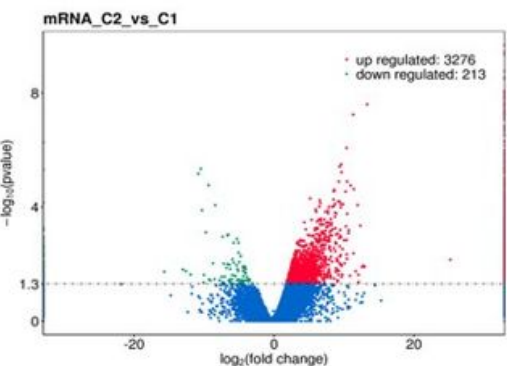

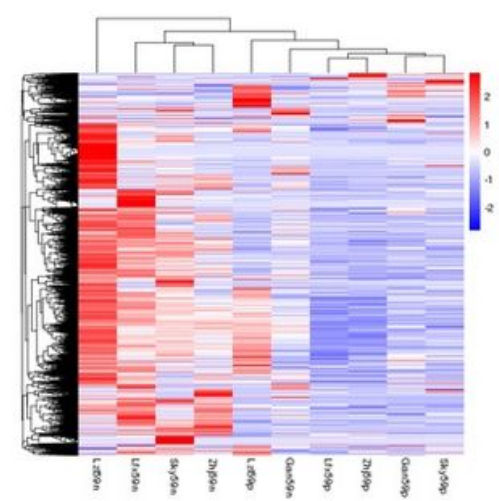

$\mathrm{E}$

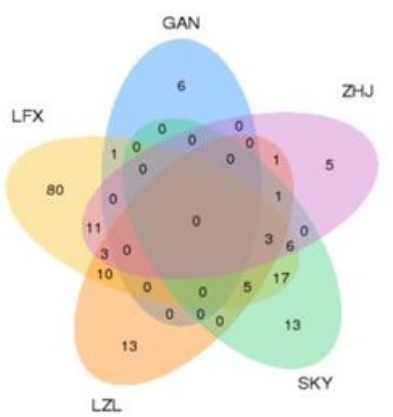

D
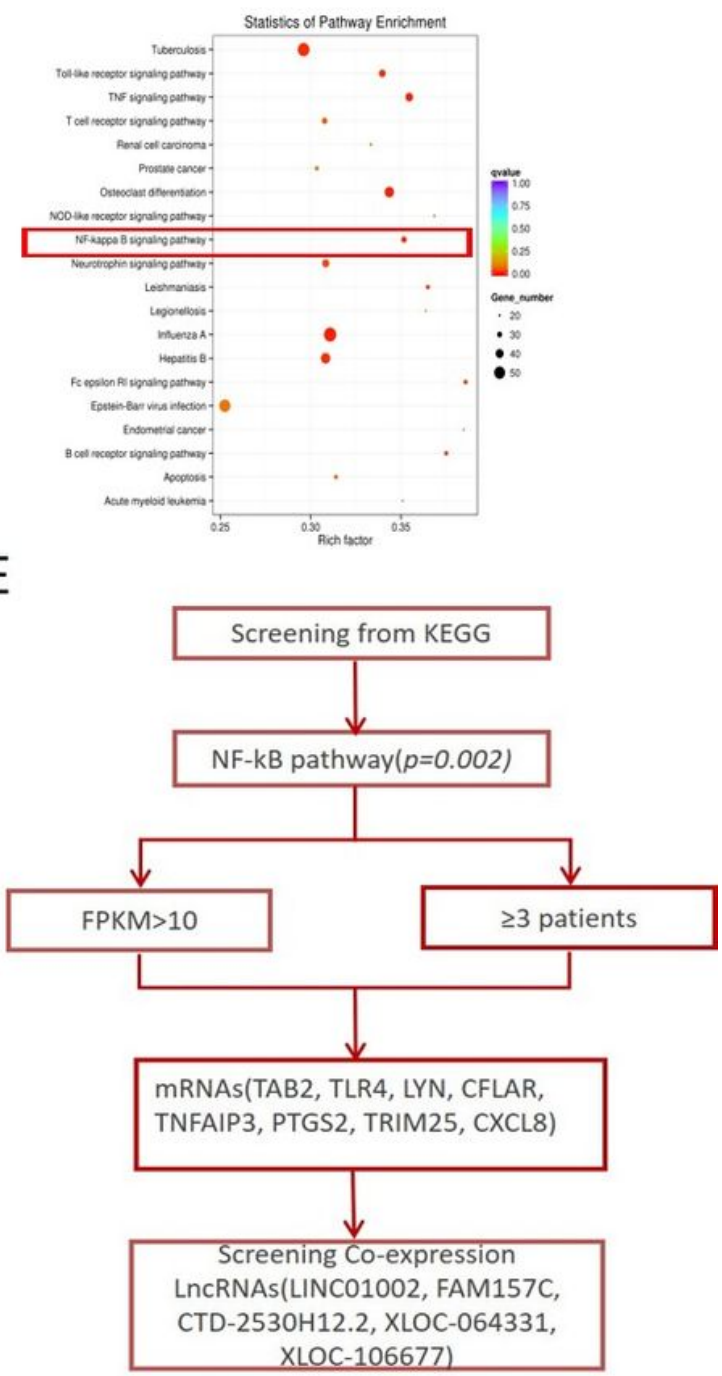

Figure 2

A. Volcanic map of differentially expressed LncRNAs and mRNAs, C1 represents CD59+ cells, and C2 represents CD59- cells; B. Heap map of differentially expressed LncRNAs and mRNAs; C. Venn diagrams of upregulated LncRNAs and mRNAs in $5 \mathrm{PNH}$ patients. 4 mRNAs expressed in 5 patients, $118 \mathrm{mRNAs}$ expressed in 4 patients, 335 mRNAs expressed in 3 patients, 3 LncRNAs expressed in 4 patients, 15 LncRNAs expressed in 3 patients; D. Scatter plot is a graphical representation of KEGG enrichment analysis results. In this figure, KEGG enrichment was measured by rich factor, q-value and the number of genes enriched in this pathway. The greater the Rich factor, the greater the degree of enrichment. Q-value is the p-value after multiple hypothesis testing and correction. The value range of q value is $[0,1]$. The closer to zero, the more significant the enrichment; E. Screening of mRNAs and LncRNAs from NF-kB pathway. 
A

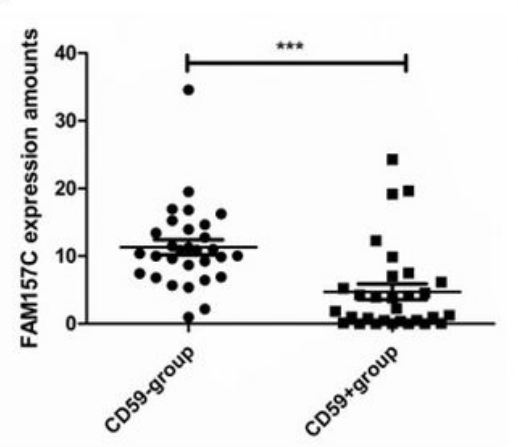

D

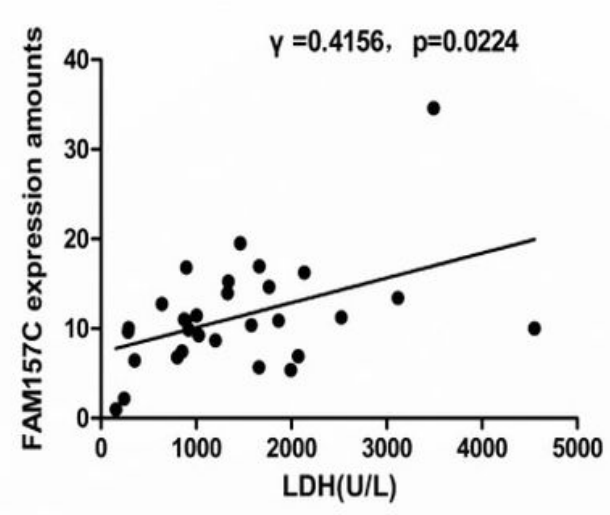

C

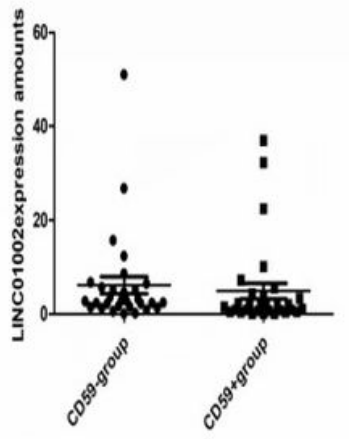

CD59- group

$\mathbb{Q} \mathrm{CD}^{\circ} 9^{+}$group
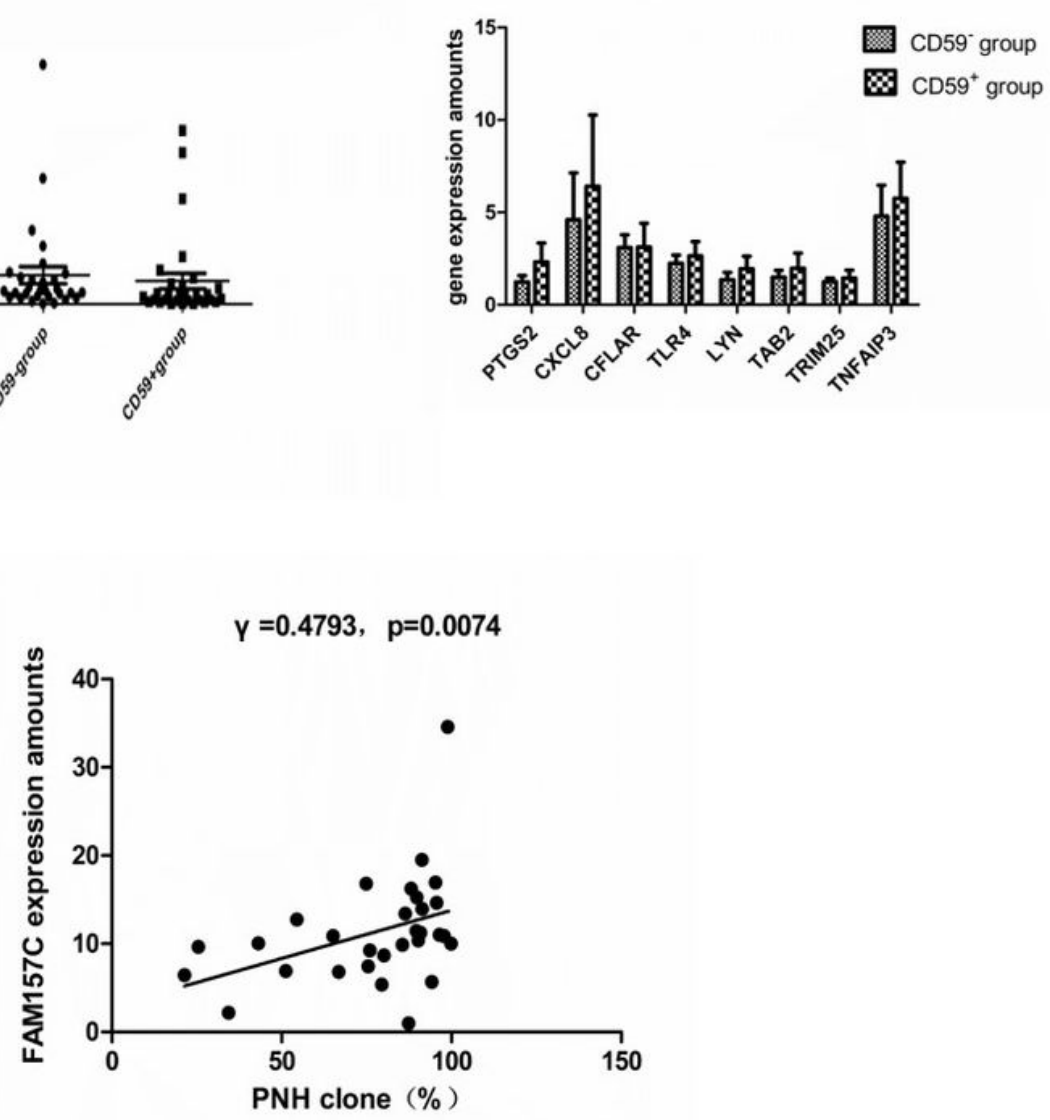

Figure 3

A. The expression of FAM157C in 30 PNH patients; B. The expression of LINC01002 in 30 PNH patients; C. The expression of mRNAs in $30 \mathrm{PNH}$ patients; D. Correlation analysis between FAM157C expression and clinical date. The expression level of FAM157C were positive correlation with LDH level and CD59- granulated and monocytes cells ratio. 


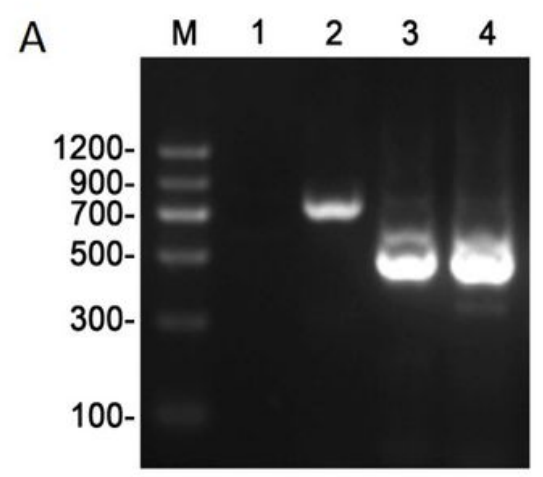

B

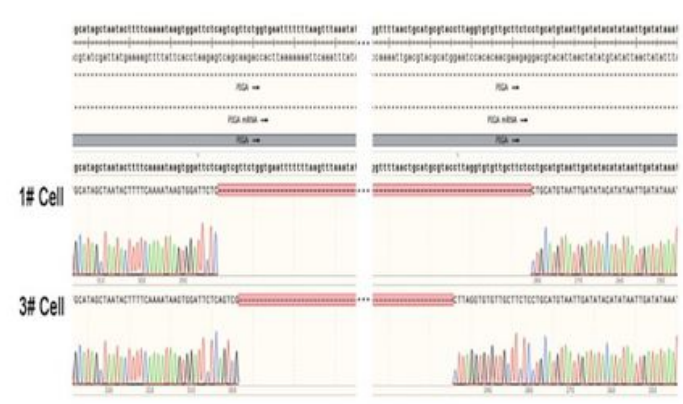

C
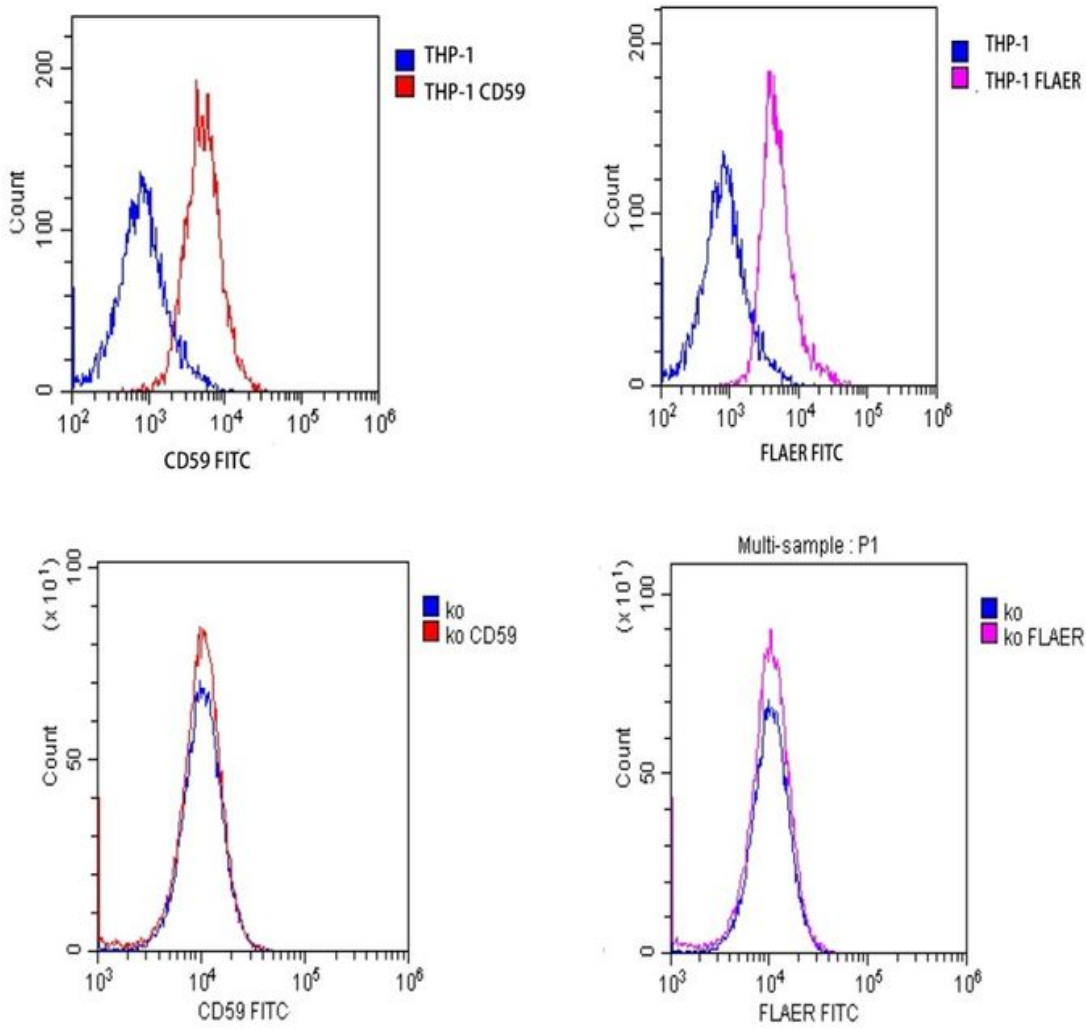

Figure 4

PCR identification of PIGA-KO-THP-1 monoclonal cells. M: DL-1200, Lane-2: Negative Control, Lane-3: THP-1 WT Cell, Lane-4: 1\# THP-1 KO cell, Lane-5: 3\# THP-1 KO cell. B. Gene sequencing results of PIGA-KO-THP-1 monoclonal cells. 1 \# and $3 \#$ cell: THP-1 KO cell. C. Surface expression of GPI-APs on THP-1 cells and PIGAKO-THP-1 cells. Test cells were stained with FLAER (purple lines) and anti-CD59 (red lines) antibody. Negative staining controls (blue lines) for FLAER and anti-CD59 were buffer only and isotype matched monoclonal antibody, respectively. The expressions of GPI-APs on PIGA-KO cells were lost. 
A
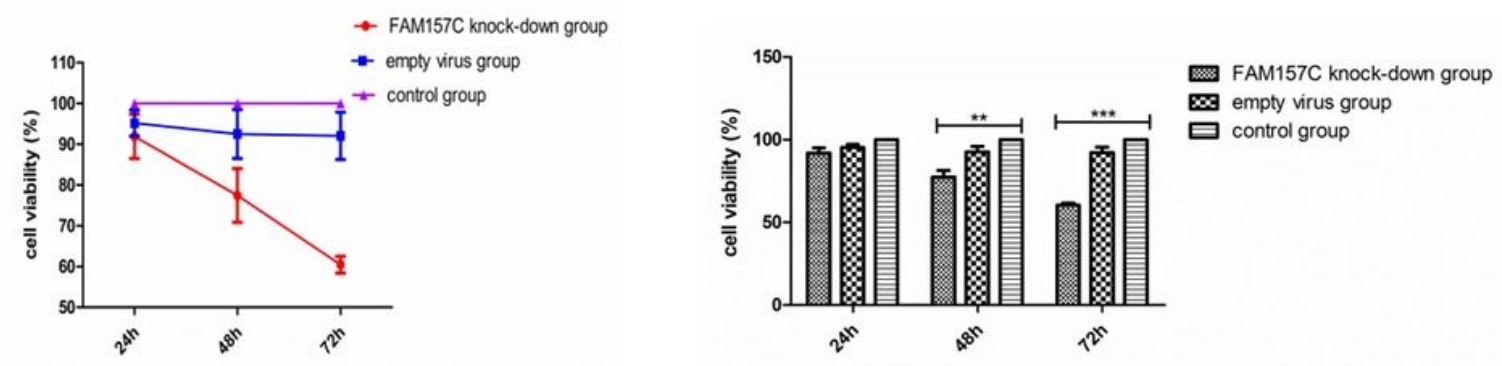

B

C
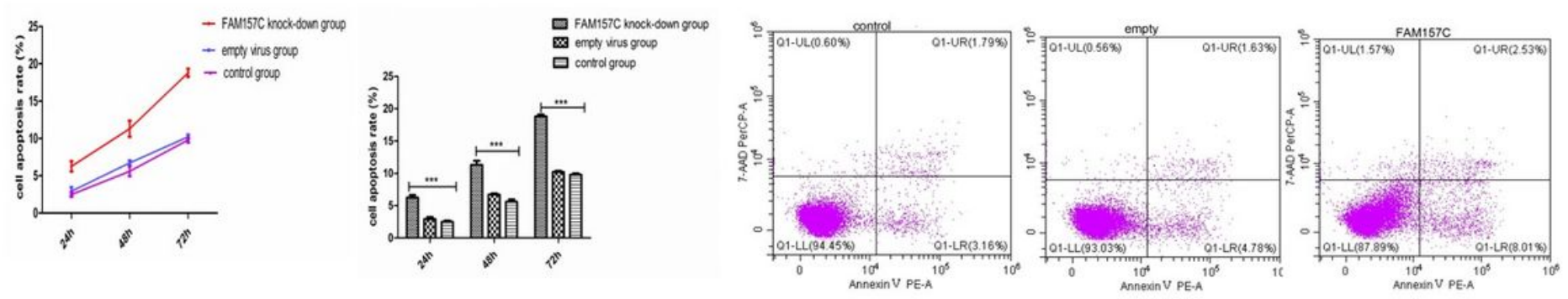

D

$E$
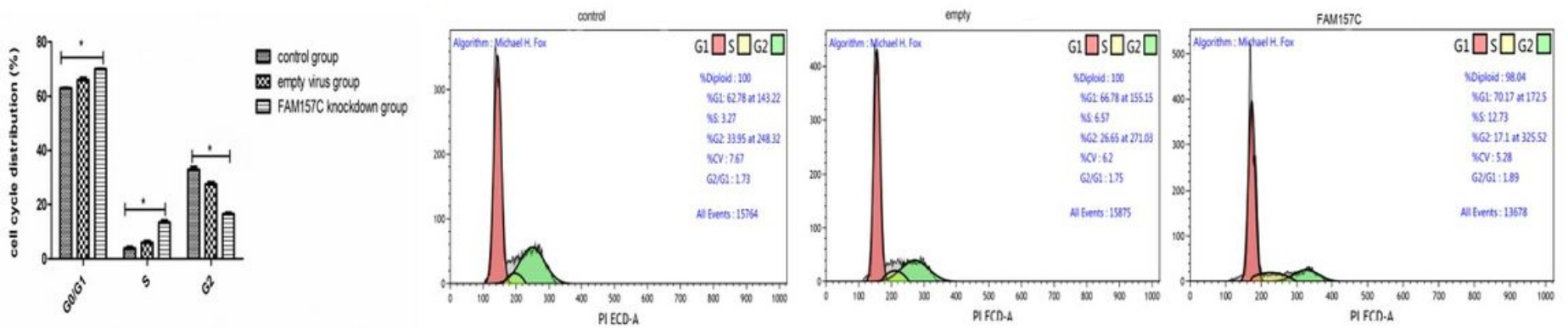

\section{Figure 5}

A. Significant decreased proliferation ability by FAM157C knock down was detected after transfection, compared with control group and empty virus transfection group; B. Significantly increased cell apoptosis rate by FAM157C knock down was observed after transfection, compared with control group and empty virus transfection group; $\mathrm{C}$. The cell apoptosis rate was examined by flow cytometry; $\mathrm{D}$. The cell cycle phase assay after transfection, the percentage of G2-phase post-replicating cells in FAM157C knock down group significantly reduced, while the percentage of G0/G1 phase and S phase cells significantly increased, compared to control group and empty virus transfection group. E. The cell cycle phase was examined by flow cytometry.

\section{Supplementary Files}

This is a list of supplementary files associated with this preprint. Click to download.

- Appendices1.doc

- Appendices2.doc 